\title{
Identification of protein kinase SNF1 in CitEST
}

\author{
Simone Guidetti-Gonzalez, Carlos Francisco Ragassi and Helaine Carrer \\ Departamento de Ciências Biológicas, Escola Superior de Agricultura Luiz de Queiroz, \\ Universidade de São Paulo, Piracicaba, SP, Brazil.
}

\begin{abstract}
SnRKs (Sucrose non-fermenting-1 related kinases) is a family of protein kinases found in many crops, such as Arabidopsis, rice, sugarcane, tomato and several other plant species. This family of proteins is also present in other organisms like Saccharomyces cerevisiae (sucrose non-fermenting-1 - Snf1) and in mammals (AMP-activated protein kinases - AMPKs). There is evidence that SnRKs play an important role in plant responses to nutritional and environmental stresses and that SnRKs also play a major role in controlling key enzymes in the biosynthetic pathways of plants. In this work, we identified 18 contigs and two singletons encoding putative SnRKs in the CitEST database. All of them present highly conserved N-terminal catalytic domain, which is found in the SnRKs families of several plant species. Through comparison with known SnRKs, we were able to classify them into three subfamilies.
\end{abstract}

Key words: SnRK, sucrose non-fermenting-1 (SNF1), transcription regulator.

Received: July 21, 2006; Accepted: April 17, 2007.

Snfl (sucrose non-fermenting-1) is a protein family that plays a major role in regulating gene expression in eukaryotic cells. This family of regulatory proteins is represented by sucrose non-fermenting-1 (SNF1) protein kinase in Saccharomyces cerevisiae, AMP-activated protein kinases (AMPKs) in mammals and SnRKs (Sucrose non-fermenting-1-related protein kinases) in higher plants (Carraro et al., 2001).

The first protein of Snf1 class to be characterized was from Saccharomyces cerevisiae (Carlson et al., 1981; Celenza and Carlson, 1984). In this organism, the Snf1 protein is required for stress responses, notably in the adaptation of cells to carbon stress. It also regulates the transcription of many genes in response to glucose limitation and is required for utilization of alternate carbon sources (Hong et al., 2003). Members of the AMP-activated/SNF1-related protein kinase subfamily are central components of highly conserved protein kinase cascades that now appear to be present in most, if not all, eukaryotic cells (Hardie et al., 1998). The SnRKs have been associated with cellular processes involved in responses to nutritional and environmental stresses that deplete cellular ATP levels in higher plants (Hardie, 1999; Sugden et al., 1999). Plant SnRKs regulate the activity of rate limiting metabolic enzymes, including 3-hydroxy-3-methylglutaryl-CoA reductase, nitra-

Send correspondence to Helaine Carrer. Departamento de Ciências Biológicas, Escola Superior de Agricultura Luiz de Queiroz, Universidade de São Paulo, Av. Pádua Dias 11, 13418-900 Piracicaba, SP, Brazil. E-mail: hecarrer@esalq.usp.br. te reductase, and sucrose phosphate synthase (Sugden et al., 1999), as well as the transcription of glucose and stress-regulated genes (Purcell et al., 1998; Bhalerao et al., 1999).

Based on the sequences of their kinase domains that are constituted by N-terminal serine/threonine kinase domain and a C-terminal regulatory domain (Celenza and Carlson, 1986; Gancedo, 1998), the Snf1 family is considered a distinct subgroup of the protein kinase superfamily (Halford and Hardie, 1998). During evolution, the family of plant Snf1-related protein kinases has expanded and split into three subfamilies: SnRK1a/1b, SnRK2, and SnRK3. The SnRK1 subfamily represents direct structural and functional homologues of the SNF1/AMPK family (Halford and Hardie, 1998). SnRK1 homologs from various plant species can complement yeast snfl defective mutant phenotypes, suggesting an evolutionary conservation in its function (Rolland et al., 2006). Schwachtje et al. (2006) found that the $\beta$-subunit of an SnRK1, GAL83, regulates a herbivore-induced response in Nicotiana attenuata, which increases the allocation of sugar to roots. The transcripts of GAL83 are rapidly down regulated in source leaves after herbivore attack and, when silenced, increase transport of assimilates to roots. This result demonstrates that SnRK1 can alter resource allocation, improving plant tolerance to herbivory. The SnRK2 and SnRK3 groups are unique to plants and may be involved in the response to environmental stresses (Kelner et al., 2004). Although SnRK2 and SnRK3 are clearly within the SNF1 class, they are signifi- 
cantly less similar to SNF1 and AMPK when compared to SnRK1; SnRK2s and SnRK3s have only 42\%-45\% amino acid sequence identity to SnRK1, SNF1 and AMPK in the catalytic domain (Halford et al., 2004).

The SnRK1b group appears to be present only in cereals whereas the SnRK1a group appears to be present in both dicotyledonous and monocotyledonous plants (Zhang et al., 2001). In the Arabidopsis genome, only three members of the SnRK1 family were identified (Hrabak et al., 2003). The SnRK2 subfamily includes PKABA1 from wheat, which is involved in mediating ABA-induced changes in gene expression, including ABA suppression of gibberellic acid signaling in the aleurone layers (GomezCadenas et al., 1999; Gomez-Cadenas et al., 2001). The results obtained by Kobayashi et al. (2004) indicate that the rice SnRK2 protein kinase family has evolved specifically for hyper-osmotic stress signaling and individual members have acquired distinct regulatory properties, including ABA responsiveness by modifying the $\mathrm{C}$-terminal domain. Both Arabidopsis and rice genomes encode 10 members of the SnRK2 protein kinase family (Hrabak et al., 2003; Kobayashi et al., 2004).

Several kinases of the SnRK3 subfamily have been described and one is the wheat (Triticum aestivum) protein kinase WPK4, whose expression is up-regulated by light, cytokinins and low temperature, and it is down-regulated by sucrose (Ikeda et al., 1999). There are several reports indicating that a group of kinases interact with calcineurin B-like proteins that belong to SnRK3 subfamily and take part in protection of plant cells against abiotic stresses (Luan et al., 2002; Chinnusamy et al., 2004; Gong et al., 2004). The best-known member of this group is the Arabidopsis SOS2 protein kinase, which is required for sodium and potassium ion homeostasis and salt tolerance (Liu et al., 2000). Analysis of the Arabidopsis genome identified 25 members of the SnRK3 family (Halford et al., 2003). The similarity of the catalytic domains of the SnRK1, 2 and 3 subfamilies of protein kinases suggest that they could have a degree of overlap in substrate specificity and function (Halford and Hardie, 1998).

Carraro et al. (2001) identified, in the SUCEST database (Vettore et al., 2001), 22 sugarcane EST-contigs encoding putative SnRKs based on analysis of 26 cDNA libraries; the authors suggested that sugarcane SnRKs might be involved in the development of plants and their responses to the environment.

This work identified, in the CitEST (Citrus EST) database (http://biotecnologia.centrodecitricultura.br/), sequences with a high degree of similarity to SNF1 and SnRKs. We used the deduced amino acid sequence of the $S$. cerevisiae Snf1 (accession number M13971), a SNF1-like protein kinase from Arabidopsis thaliana (BAB85674) and Lycopersicon esculentum (AAF66639). The search was performed using the T-BLASTN program (Altschul et al., 1990) selecting reads with E-values lower than $10^{-10}$ that were analyzed to form contigs and singletons by CAP3 Program (Contig Assembly Program-Third generation) (Huang and Madan, 1999).

Based on amino acid sequence, we identified 535 reads similar to SNF1 and SnRKs $\left(\mathrm{e} \leq 10^{-10}\right)$, which formed 108 contigs and 78 singletons; from those, 18 contigs and two singletons showed a very conserved N-terminal catalytic domain and these were used for further analysis. Sequences of the conserved N-terminal domain were compared using the AlignX program (Vector NTI Program, InforMax, Inc., default parameters), with SnRKs sequences from Arabidopsis thaliana, Lycopersicon esculentum and Oryza sativa obtained from NCBI Genbank. The alignments showed a highly conserved N-terminal catalytic domain, including the conserved T-loop motif (Figure 1). The average size of the contigs were: 200-300 amino acids long for three contigs; 301-400 for five contigs and two singletons; 401-500 for five contigs and five contigs that were more than 500 amino acids in length. The best hits by the BLAST search to homologous organisms are shown in Table 1 .

The NCBI database (http://www.ncbi.nlm.nih.gov/) has 184,881 and 28,822 nucleotide sequences of Citrus and Poncirus species, respectively. Searches in this database did not identify sequences related to SNF1 or SnRKs. According to these data, this work can be considered the first attempt to catalog this family of proteins in Citrus and Poncirus species. In addition to Halford and Hardie (1998) and Carraro et al. (2001), our phylogenetic analysis, generated by the AlignX program (Vector NTI Program, InforMax, Inc., default parameters), also showed that yeast Snf1 formed a different clade. However, with a closer relationship to the SnRK1 subfamily (Figure 2), as reported by Halford and Hardie (1998) and in contrast to Carraro et al. (2001), the SnRK1 subfamily was shown to be more closely related to SnRK2 than to the SnRK3 subfamily (Figure 2). These results were expected because during the evolutionary divergence of the SnRK family, the SnRK2 proteins played an intermediate position. SnRK2s are involved in mediating ABA-induced changes in gene expression and in osmotic stress signaling in plants (Kobayashi et al., 2004; Boudsocq et al., 2007). SnRK1s are activated by ABA (Bradford et al., 2003; Weber et al., 2005) and SnRK3s show specific roles in plant adaptation to osmotic stresses (Liu et al., 2000), leading the SnRK2 family to occupy the intermediate position in the phylogenetic tree, between the SnRK1 and SnRK3.

With the comparison of amino acid sequences of CitEST contigs and singletons encoding putative SnRKs to known SnRKs from NCBI database, we were able to classify the SnRKs into different subfamilies: one in SnRK1 (SnRK1a subfamily), four in SnRK2 (two in SnRK2a and two in SnRK2b) and fifteen in SnRK3 (Figure 2). In our analysis, no contigs or singletons that are related to the SnRK1b subfamily were identified, suggesting that this 


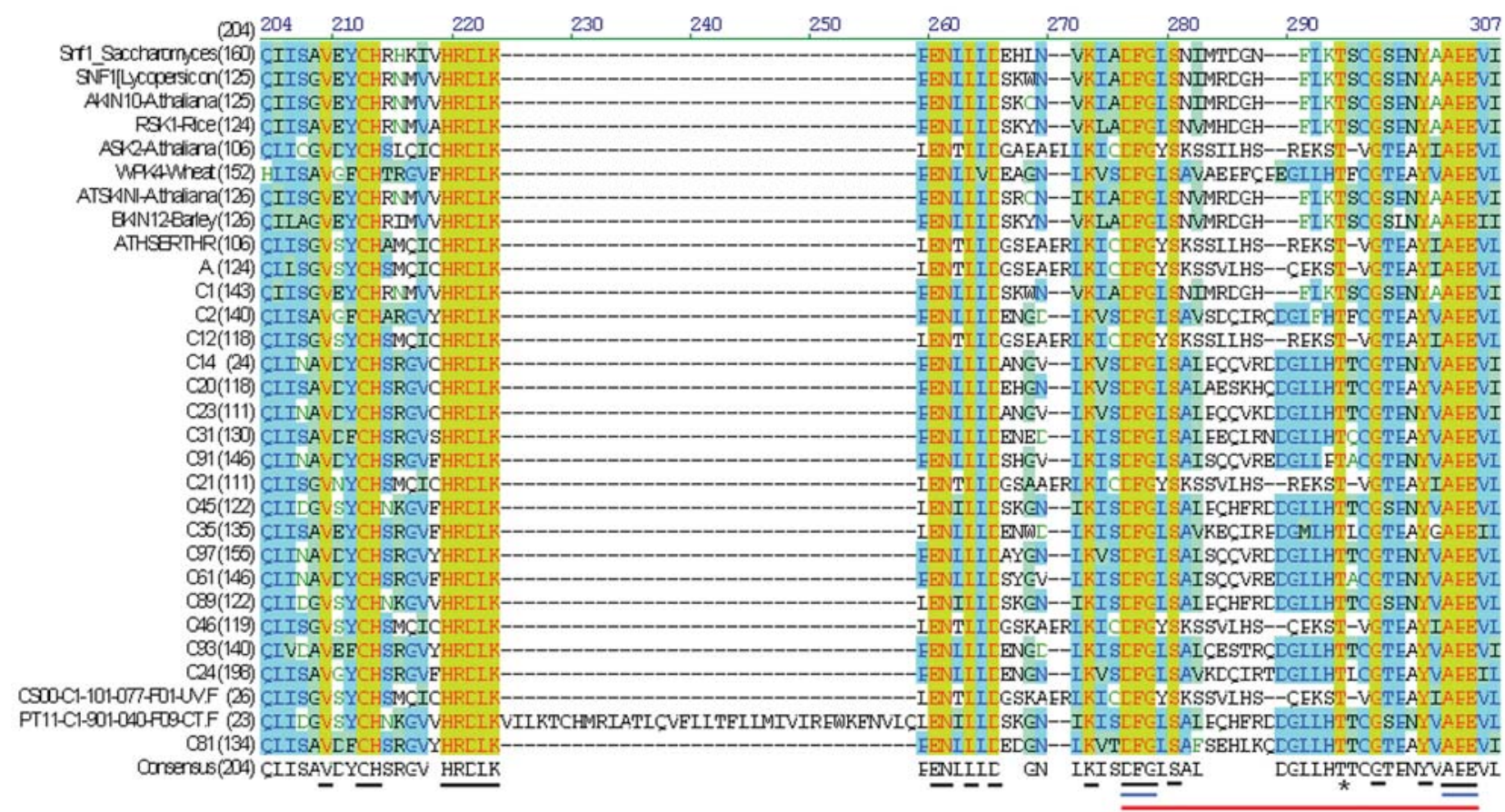

Figure 1 - Alignment of part of the N-terminal catalytic domain and the T-loop domain of CitEST contigs and singletons encoding putative SnRKs, SNF1 and other SnRKs. Accession numbers are as follows: SNF1 - Saccharomyces cerevisiae (M13971); SNF1 - Lycopersicon esculentum (AAF66639); AKIN10 - A. thaliana (M93023); RSK1 - Rice (U55768); ASK2 - A. thaliana (Z12120); WPK4 - Wheat (D21204); ATSKINI - A. thaliana (X94755); BKIN12 - Barley (X65606); ATHSERTHR (M91548); and A (A. thaliana) (S71172). Amino acids highlighted in yellow are identical in all the proteins. Blue represents consensus residues derived from a block of similar residues; green represents consensus residues derived from the occurrence of more than $50 \%$ of a single residue at a given position. The conserved T-loop domain is underlined in red. The DFG and APE conserved T-loop boundary consensi are underlined in blue. The putative phosphorylated threonine is indicated by asterisk $(*)$.

family might be specific for grasses. This statement is in agreement with Harford and Hardie (1998) where the SnRK1b subfamily is present in rice, oat, rye and barley.

The SNF1, AMPKs and SnRKs are regulated by phosphorylation on a threonine (equivalent to Thr172 on mammalian AMPK and to Thr175 in Arabidopsis thaliana SnRK1 - AKIN10), within the "T-loop" between the conserved DFG and APE motifs (Sugden et al., 1999). The T-loop motif is present in all putative CitEST SnRKs, suggesting conserved activities (Figure 1).

The SNF1 from Lycopersicon esculentum (LeSNF1) is constitutively expressed in both, seeds and leaves regardless of the developmental, hormonal, or environmental conditions (Bradford et al., 2003). In the CitEST database, we found one contig $(\mathrm{C} 1)$ highly similar to this gene (Table 1), which also belongs to the SnRK1 family (Figure 2) and is represented by 17 reads from leaf (11 reads) and fruit (6 reads) libraries, showing a non-specific pattern of expression. Maybe a constitutive expression can be suggested due to the presence of reads from different stages of fruit development and reads from healthy and pathogen infected leaf libraries.

The osmotic stress-activated protein kinase (NtOSAK) from Nicotiana tabacum is involved in stress signaling pathways in plants and represents the first characterized SnRK2 protein (Kelner et al., 2004). In our analy- ses, we identified two contigs, whose deduced amino acid sequences are highly similar to this protein (Table 1) and in the phylogenetic analysis these contigs also belong to the SnRK2 family (SnRK2b subfamily) (Figure 2). One of these contigs $(\mathrm{C} 21)$ presents two reads from $C$. sinensis fruit libraries and the other contig $(\mathrm{C} 12)$ is formed by three reads from $C$. sinensis fruit libraries, one from C. reticulata fruit library and one from $C$. sinensis leaf library, showing a tendency of expression in the beginning of the fruit development in these species. The lack of a specific library prepared under osmotic stress in the CitEST database makes it difficult to relate any protein to this abiotic stress, so we can just suggest the possible involvement of these putative proteins present in citrus fruit development.

The rice genome has at least 10 SnRK2 protein kinases, which are all activated by hyperosmotic stress, and three of them are also activated by ABA (Kobayashi et al., 2004). These protein kinases were designated SAPK1 through SAPK10. Whereas all the members of the rice SnRK2 family are activated by $\mathrm{NaCl}$ treatment, only SAPK8, SAPK9, and SAPK10 are activated by ABA. Treatments with $\mathrm{ABA}(50 \mu \mathrm{M}), \mathrm{NaCl}(150 \mathrm{mM})$, or mannitol $(600 \mathrm{mM})$ show that rice SAPK1 is up-regulated by all these treatments in both, root and the above-ground organs. Although the effect of ABA was weaker than those of the other two treatments, SAPK1 is highly homologous to 
Table 1 - Numbers of contigs and singletons with similarity to SnRK genes.

\begin{tabular}{|c|c|c|c|c|c|}
\hline Gene & Homologous organism & Accession number & Number of contigs & Number of singletons & Best $e$-value \\
\hline \multicolumn{6}{|l|}{ SnRK1 } \\
\hline LeSNF1 & Lycopersicon esculentum & AAF66639 & 1 & 0 & 0.0 \\
\hline \multicolumn{6}{|l|}{ SnRK2 } \\
\hline NtOSAK & Nicotiana tabacum & AAL89456 & 2 & 0 & $2 e-166$ \\
\hline SAPK1 & Oryza sativa & Q75LR7 & 1 & 0 & $7 e-128$ \\
\hline PKABA1 & Oryza sativa & AAO65504 & 0 & 1 & $6 e-50$ \\
\hline \multicolumn{6}{|l|}{ SnRK3 } \\
\hline OsPK4 & Oryza sativa & BAA 83688 & 1 & 0 & $2 \mathrm{e}-130$ \\
\hline SOS 2 & Arabidopsis thaliana & AAK26847 & 1 & 0 & 0.0 \\
\hline SOS2 & Arabidopsis thaliana & O22932 & 1 & 0 & $6 e-107$ \\
\hline SOS 2 & Glycine $\max$ & AAM83095 & 1 & 0 & $1 \mathrm{e}-160$ \\
\hline$Z m P K 4$ & Zea mays & AAF22219 & 1 & 0 & $5 e-96$ \\
\hline wpk4 & Arabidopsis thaliana & AAK62444 & 7 & 0 & $5 \mathrm{e}-143$ \\
\hline wpk4 & Arabidopsis thaliana & AAM13241 & 1 & 1 & e-98 \\
\hline wpk4 & Oryza sativa & BAD73090 & 1 & 0 & $2 \mathrm{e}-60$ \\
\hline
\end{tabular}

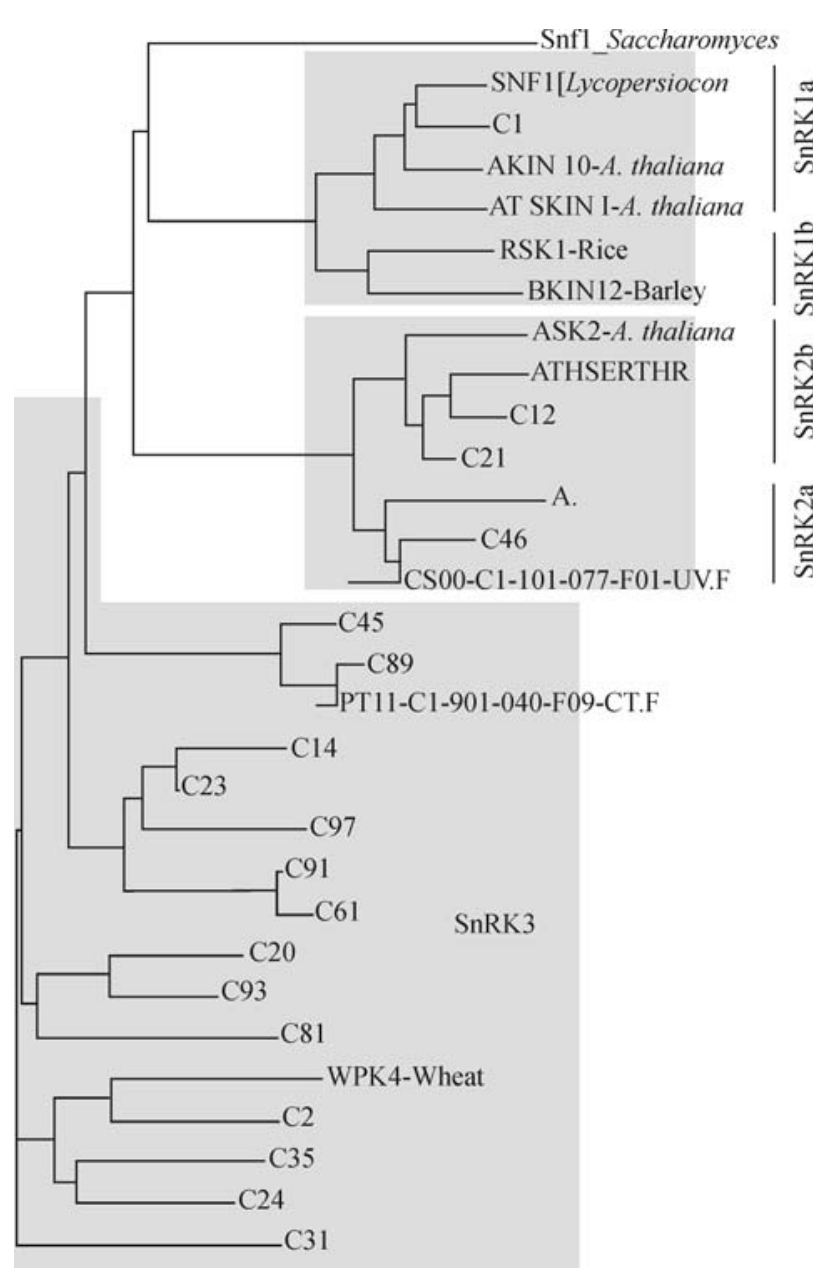

Figure 2 - Phylogenetic relationships between members of the SnRK families and SNF1. Alignment of N-terminal catalytic and T-loop domain amino acid sequences was performed using AlignX (Vector NTI program). SnRKs subfamilies are boxed in gray. wheat PKABA1 (that is abcisic acid-inducible protein). SAPK1 requires a higher level of stress for activation (Kobayashi et al., 2004). We found one contig similar to SAPK1 and one singleton (CS00-C1-101-077-F01-UV.F) similar to rice PKABA1 protein (Table 1), also shown in the phylogenetic tree (Figure 2) as part of the SnRK2 family. Further studies are necessary to confirm the functions of these proteins in the resistance to osmotic stress and in ABA-induced changes in gene expression, as described by Kobayashi et al. (2004).

The Triticum aestivum gene WPK4 encodes a $56-\mathrm{kDa}$ protein kinase that belongs to group 3 of the SNF1-related protein kinase family (SnRK3). It is up-regulated by light and cytokinins and down-regulated by sucrose (Sano and Youssefian, 1994). The Oryza sativa gene OsPK4 encodes a protein with a $75 \%$ sequence similarity to WPK4 (Ohba et al., 2000). Transcripts of OsPK4 were detected in all tissues tested, and the amount increased upon illumination, nutrient deprivation and treatment with cytokinins (Ohba et al., 2000). A Zea mays gene, $Z m P K 4$, encodes a protein with $74 \%$ similarity to WPK4. Its transcripts were constitutively expressed in all tissues, regardless of light, nutrient and cytokinin status, but were increased upon exposure to low temperature (Ohba et al., 2000). In our analysis, we identified one contig similar to ZmPK4 (C93), one contig (C20) related to OsPK4, one contig (C35) similar to the Oryza sativa WPK4 homologue protein, and eight contigs and one singleton (PT11-C1-901-040-F09-CT.F) that is highly similar to Arabidopsis thaliana WPK4 homologue. This singleton has a 35 amino acid insertion downstream from the conserved T-loop motif. Such an insertion is not found in any other putative SnRK (Figure 1). Four of these contigs that are similar to Arabidopsis thaliana WPK4 show a specific pattern of expression. Two of them show strong ten- 
dency to highly express in fruit; the contigs 14 (6e-110) and 23 (e-109) present 8 and 5 reads, respectively. They are derived from C. sinensis fruit libraries. Meanwhile, contigs 89 (3e-98) and 91 (3e-88) show a tendency of expression in $P$. trifoliata Citrus tristeza virus-infected leaf, suggesting a possible role in plant-pathogen interaction.

Among the contigs related to SOS2 protein, only one is formed by reads from a specific library. Contig 81 is highly related to the SOS2 protein of Glycine max, with tendency of expression in C. reticulata fruit libraries (11 reads), and may play a role in fruit development in citrus plants.

Little is known about the physiological role of SnRK in plants when compared to yeast and mammals. The data suggest the involvement of this plant kinase complex in the global regulation of metabolism as well as in developmental and stress responses (Polge and Thomas, 2007). Members of SnRK subfamilies are differently regulated in response to environmental stimuli, such as light, nutrient deprivation and cytokinins (Ohba et al., 2000). It is induced in the early stages of seed development in rice (Takano et al., 1998), whereas there is a SnRK from rice (REK - rice endosperm kinase) that is induced specifically in leaves and maturing seeds but not in stem and roots (Hotta et al., 1998), suggesting that plant SnRKs might have a function in tissue or organ development. Besides, the reads belonging to the contigs related to different SnRKs from various libraries such as leaves from $C$. aurantium, $C$. sinensis, $C$. reticulata, Poncirus trifoliata and fruits from C. sinensis and $C$. reticulata suggest a non-specific pattern of expression; and, in the same cases, we can infer a constitutive expression. Since the CitEST database lacks sequences from libraries prepared from tissues under osmotic stress, we cannot infer the relation of the putative SnRKs responses identified to this specific condition. Data described here, also suggest the involvement of the putative SnRKs in fruit developmental and plant-pathogen interaction responses, although further studies on the expression and specific functions of these identified genes will be necessary to a better understanding of the genetic and biochemical basis of SnRKs in citrus plants.

\section{Acknowledgments}

The authors would like to thank CNPq/ Millennium Institute (62.0054/01-8) for financially supporting the CitEST project, FAPESP for financial support provided to Simone Guidetti-Gonzalez (05/58117-4) and PIBIC/CNPq (100037/2004-6) for financial support provided to Carlos Francisco Ragassi.

\section{References}

Altschul SF, Gish W, Miller W, Myers EW and Lipman DJ (1990) Basic local alignment search tool. J Mol Biol 215:403-410.

Bhalerao RP, Salchert K, Bakó L, Ökrész L, Szabados L, Muranaka T, Machida Y, Schell J and Koncz C (1999) Regulatory interaction of PRL1 WD protein with Arabdopsis SNF1-like protein kinases. Proc Natl Acad Sci USA 96:5322-5327.

Boudsocq M, Droillard MJ, Barbier-Brygoo H and Lauriere C (2007) Different phosphorylation mechanisms are involved in the activation of sucrose non-fermenting 1 related protein kinases 2 by osmotic stresses and abscisic acid. Plant Mol Biol 63:491-503.

Bradford KJ, Downie AB, Gee OH, Alvarado V, Yang H and Dahal P (2003) Abscisic acid and gibberellin differentially regulate expression of genes of the SNF1-related kinase complex in tomato seeds. Plant Physiol 132:1560-1576.

Carlson M, Osmond BC and Botstein D (1981) Mutants of yeast defective in sucrose utilization. Genetics 98:25-40.

Carraro DM, Lambais MR and Carrer H (2001) In silico characterization and expression analyses of sugarcane putative sucrose non-fermenting-1 (SNF1) related kinases. Genet Mol Biol 24:35-41.

Celenza JL and Carlson M (1984) Cloning and genetic mapping of SNF1, a gene required for expression of glucose-repressible genes in Saccharomyces cerevisiae. Mol Cell Biol 4:49-53.

Celenza JL and Carlson M (1986) A yeast gene that is essential for release from glucose repression encodes a protein kinase. Science 233:1175-1180.

Chinnusamy V, Schumaker K and Zhu J-K (2004) Molecular genetic perspectives on cross-talk and specificity in abiotic stress signaling in plants. J Exp Bot 55:225-336.

Gancedo JM (1998) Yeast carbon catabolic repression. Microbiol Mol Biol Rev 62:341-361.

Gomez-Cadenas A, Verhey SD, Holappa LD, Shen Q, Ho T-HD and Walker-Simmons MK (1999) An abscisic acid-induced protein kinase, PKABA1, mediates abscisic acid-suppressed gene expression in barley aleurone layers. Proc Natl Acad Sci USA 96:1767-1772.

Gomez-Cadenas A, Zentella R, Walker-Simmons MK and Ho T-HD (2001) Gibberelin/abscisic acid antagonism in barley aleurone cells: Site of action of the protein kinase PKABA1 in relation to gibberellin signaling molecules. Plant Cell 13:667-679.

Gong D, Guo Y, Schumaker KS and Zhu J-K (2004) The SOS3 family of calcium sensors and SOS2 family of protein kinases in Arabidopsis. Plant Physiol 134:919-926.

Halford NG and Hardie DG (1998) SNF1-related protein kinases: Global regulators of carbon catabolism in plants? Plant Mol Biol 37:735-748.

Halford NG, Hey S, Jhurreea D, Laurie S, McKibbin RS, Paul M and Zhang Y (2003) Metabolic signalling and carbon partitioning: Role for Snf1-related (SnRK1) protein kinase. J Exp Bot 54:467-475.

Halford NG, Hey S, Jhurreea D, Laurie S, McKibbin RS, Zhang Y and Paul MJ (2004) Highly conserved protein kinases involved in the regulation of carbon and amino acid metabolism. J Exp Bot 55:35-42.

Hardie DG (1999). Roles of the AMP-activated/SNF1 protein kinase family in the response to cellular stress. Biochem Soc Sym 64:13-27.

Hardie DG, Carling D and Carlson M (1998) The AMP-activated/SNF1 protein kinase subfamily: Metabolic sensors of the eukaryotic cell? Annu Rev Biochem 67:821-855.

Hong S-P, Leiper FC, Woods A, Carling D and Carlson M (2003) Activation of yeast Snf1 and mammalian AMP-activated 
protein kinase by upstream kinases. Proc Natl Acad Sci USA 100:8839-8843.

Hotta H, Aoki N, Matsuda T and Adachi T (1998) Molecular analysis of a novel protein kinase in maturing rice seed. Gene 213:47-54.

Hrabak EM, Chan CW, Gribskov M, Harper JF, Choi JH, Halford N, Kudla J, Luan S, Nimmo HG, Sussman MR, et al. (2003). The Arabidopsis CDPK-SnRK superfamily of protein kinases. Plant Physiol 132:666-680.

Huang X and Madan A (1999) CAP3: A DNA sequence assembly program. Genome Res 9:868-877.

Ikeda Y, Koizumi N, Kusano T and Sano H (1999) Sucrose and cytokinin modulation of WPK4, a gene encoding a SNF1related protein kinase from wheat. Plant Physiol 121:813820.

Kelner A, Pkala I, Kaczanowski S, Muszyska G, Hardie DG and Dobrowolska G (2004) Biochemical characterization of the tobacco $42-k d$ protein kinase activated by osmotic stress. Plant Physiol 136:3255-3265.

Kobayashi Y, Yamamotoa S, Minamia H, Kagayab Y and Hattoria T (2004) Differential activation of the rice sucrose nonfermenting1-related protein kinase 2 family by hyperosmotic stress and abscisic acid. Plant Cell 16:1163-1177.

Liu J, Ishitani M, Halfer U, Kim CS and Zhu J-K (2000) The Arabidopsis thaliana SOS2 gene encodes a protein kinase that is required for salt tolerance. Proc Natl Acad Sci USA 97:3730-3734.

Luan S, Kudla J, Rodriguez-Conception M, Yalovsky S and Gruissen W (2002) Calmodulins and calcinerurin B-like proteins: Calcium sensors for specific signal response coupling in plants. Plant Cell 14:S389-S400.

Ohba H, Steward N, Kawasaki S, Berberich T, Ikeda Y, Koizumi N, Kusano T and Sano H (2000) Diverse response of rice and maize genes encoding homologs of WPK4, an SNF1-related protein kinase from wheat to light nutrients, low temperature and cytokinins. Mol Gen Genet 263:359-366.

Polge C and Thomas M (2007) SNF1/AMPK/SnRK1 kinases, global regulators at the heart of energy control? Trends Plant Sci 12:20-28.
Purcell PC, Smith AM and Halford NG (1998) Antisense expression of a sucrose non-fermenting-1-related protein kinase sequence in potato results in decreased expression of sucrose synthase in tubers and loss of sucrose-inducibility of sucrose synthase transcripts in leaves. Plant J 14:195-202.

Rolland F, Gonzalez-Baena E and Sheen J (2006) Sugar sensing and signaling in plants: Conserved and novel mechanisms. Annu Rev Plant Biol 57:675-709.

Sano H and Youssefian S (1994) Light and nutritional regulation of transcripts encoding a wheat protein kinase homolog is mediated by cytokinins. Proc Natl Acad Sci USA 91:25822586.

Schwachtje J, Minchin PEH, Jahnke S, Dongen JT van, Schittko U and Balswin IT (2006) SNF1-related kinases allow plants to tolerate herbivory by allocating carbon to roots. Proc Natl Acad Sci USA 103:12935-12940.

Sugden C, Crawford RM, Halford NG and Hardie DG (1999) Regulation of spinach SNF1-related (SnRK1) kinases by protein kinases and phosphatases is associated with phosphorylation of the T loop and is regulated by 5'-AMP. Plant J 19:433-439.

Takano M, Kajiva-Kanegae H, Funatsuki H and Kikuchi S (1998) Rice has two distinct classes of kinase genes related to SNF1 of Saccharomyces cerevisiae, which are differently regulated in early seed development. Mol Gen Genet 260:388394.

Vettore AL, da Silva FR, Kemper EL and Arruda P (2001) The libraries that made SUCEST. Genet Mol Biol 24:1-7.

Weber H, Borisjuk L and Wobus U (2005) Molecular physiology of legume seed development. Annu Rev Plant Biol 56:253279.

Zhang Y, Shewry PR, Jones H, Barcelo P, Lazzeri PA and Halford NG (2001) Expression of antisense SnRK1 protein kinase sequence causes abnormal pollen development and male sterility in transgenic barley. Plant J 28:431-441.

\section{Internet Resources}

CitEST (Citrus ESTs database), http://biotecnologia. centrodecitricultura.br (April 19, 2006).

NCBI, http://www.ncbi.nlm.nih.gov/ (November 6, 2006). Associate Editor: Marco Aurélio Takita 